\title{
Evidence for Disk Photoevaporation Driven by the Central Star
}

\author{
I. Pascucci \\ Department of Physics and Astronomy, Johns Hopkins University, Baltimore, MD 21218 \\ pascucci@pha.jhu.edu \\ and \\ M. Sterzik \\ European Southern Observatory, Casilla 19001, Santiago 19, Chile \\ msterzik@eso.org
}

Received __; accepted _

^Based on observations made with VISIR on the UT3/Melipal ESO Telescope at Paranal under programme ID 080.C-0404(A) 


\begin{abstract}
The lifetime of isolated protoplanetary disks is thought to be set by the combination of viscous accretion and photoevaporation driven by stellar high-energy photons. Observational evidence for magnetospheric accretion in young sun-like stars is robust. Here we report the first observational evidence for disk photoevaporation driven by the central star. We acquired high-resolution $(\mathrm{R} \sim 30,000)$ spectra of the [Ne II] $12.81 \mu \mathrm{m}$ line from 7 circumstellar disks using VISIR on Melipal/VLT. We show that the 3 transition disks in the sample all have [Ne II] line profiles consistent with those predicted by a photoevaporative flow driven by stellar extreme UV photons. The $\sim 6 \mathrm{~km} / \mathrm{s}$ blue-shift of the line from the almost face-on disk of TW Hya is clearly inconsistent with emission from a static disk atmosphere and convincingly points to the presence of a photoevaporative wind. We do not detect any [Ne II] line close to the stellar velocity from the sample of classical optically thick (non-transition) disks. We conclude that most of the spectrally unresolved [Ne II] emission in these less evolved systems arises from jets/outflows rather than from the disk. The pattern of the [Ne II] detections and non-detections suggests that extreme UV-driven photoevaporation starts only at a later stage in the disk evolution.
\end{abstract}

Subject headings: accretion, accretion disks - infrared: stars - planetary systems: protoplanetary disks - stars: individual (TW Hya, CS Cha, T Cha, VW Cha, Sz 73, Sz 102, HD 34700) 


\section{Introduction}

It is well established that most $\sim 1$ Myr-old stars are surrounded by relatively massive optically thick dust disks. By an age of $\sim 10$ Myr only a few percent of sun-like stars still

retain an optically thick dust disk (e.g. Hernández et al. 2007) and many intermediate-mass stars might already harbor second-generation dust disks (e.g. Currie et al. 2008). This fast clearing of primordial dust is in agreement with the short formation timescales of chondrules, asteroids, and planets in the Solar System (Pascucci \& Tachibana 2009). Although much less is known about the evolution of the gas component, there are at least three observables pointing to a similarly fast dispersal timescale: a) an order of magnitude lower accretion rate for the few stars still accreting at an age of $\sim 10 \mathrm{Myr}$ in comparison to 1 Myr-old stars (Muzerolle et al. 2000; Lawson et al. 2004); b) gas mass upper limits of less than $0.1 \mathrm{M}_{\mathrm{Jup}}$ in disks around non-accreting stars (Hollenbach et al. 2005; Pascucci et al. 2006); and c) upper limits on the $\mathrm{H}_{2}$-to-dust ratio of less than 10 in two 12 Myr-old edge-on disks (Lecavelier des Etangs et al. 2001; Roberge et al. 2005). Which are the physical mechanisms clearing out primordial disks?

Models of protoplanetary disk evolution suggest that most of the disk mass is cleared out by viscous evolution (accretion of gas onto the central star) and photoevaporation driven by the central star (e.g., Clarke et al. 2001; Alexander et al. 2006b; Gorti \& Hollenbach 2009). The strongest evidence that young stars are accreting nebular gas comes from the veiling of optical and UV photospheric absorption lines which is produced by the continuum emission of an accretion shock at the stellar surface (e.g. Calvet et al. 2000). In addition, the large (few hundred $\mathrm{km} / \mathrm{s}$ ) line widths and asymmetries of permitted emission lines are found to be well reproduced by models with infalling gas via magnetospheric accretion (Muzerolle et al. 1998). In contrast to the numerous diagnostics of accretion, unambiguous diagnostics of centrally-driven disk photoevaporation are lacking. 
Here we show that the profiles of [Ne II] forbidden lines at $12.81 \mu \mathrm{m}$ from disks with dust inner holes (hereafter, transition disks) are entirely consistent with those predicted from photoevaporative disk winds (Sect. 4). These observations provide the first strong evidence in favor of centrally-driven disk photoevaporation. We also demonstrate that in disks with radially continuous optically thick dust (classical, non-transition disks) most of the [Ne II] emission detected with the Spitzer Space Telescope does not arise in a disk but likely in a jet/outflow (Sect. 51). Finally, we discuss how observations, such as those presented in this paper, can be used to identify the onset of disk photoevaporation (Sect. 6).

\section{Motivation, Observations, and Data Reduction}

The IRS spectrograph on board the Spitzer Space Telescope has recently detected the $[\mathrm{Ne}$ II] $12.81 \mu \mathrm{m}$ line toward many young protoplanetary disks as well as toward the possibly more evolved transition disks (Geers et al. 2006; Pascucci et al. 2007; Lahuis et al. 2007; Espaillat et al. 2007; Ratzka et al. 2007; Flaccomio et al. 2009; Guedel et al. 2009). Lines appear spatially and spectrally unresolved indicating that the [Ne II] emission is confined to within $\sim 1,000 \mathrm{AU}$ from the central star and lines are narrower than $\sim 500 \mathrm{~km} / \mathrm{s}$. Models of disks irradiated by stellar X-rays or extreme UV (EUV, $h \nu>$ $13.6 \mathrm{eV}$ ) photons can sufficiently ionize the disk surface to reproduce the observed line fluxes

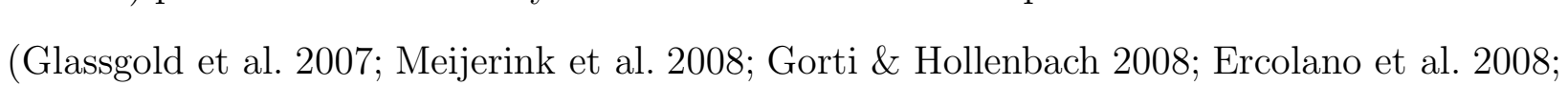
Hollenbach \& Gorti 2009) suggesting that [Ne II] emission is a robust tracer of disk gas. However, the first two spectrally resolved [Ne II] lines brought some surprises. In the case of TW Hya, Herczeg et al. (2007) found that the [Ne II] line is centered at the stellar radial velocity, consistent with a disk origin, but about a factor of two broader than that predicted from X-ray and EUV irradiated disks. van Boekel et al. (2009) spatially and spectrally resolved the strong [Ne II] line from the T Tau complex demonstrating that in this system 
most of the [Ne II] emission originates in jets/outflows with neon atoms partly ionized by stellar X-rays.

Our aim here is to enlarge the sample of spectrally resolved [Ne II] lines and understand in which systems [Ne II] emission originates in a disk. In the following subsections we describe our observational campaign and the data reduction (Sects. 2.1, 2.2, 2.3). Observations were carried out with the high-resolution ( $\mathrm{R} \sim 30,000)$ spectrograph VISIR mounted on the VLT telescope Melipal (Sect. 2.2 for more details). In addition to the already published Spitzer/IRS spectra, we reduced and present here new archival IRS spectra that aid the interpretation of the VISIR observations (Sect. 2.3).

\subsection{Target Selection}

Because of the lower-sensitivity of VISIR with respect to Spitzer/IRS, we restricted our sample to disks with bright/unresolved [Ne II] lines (Spitzer fluxes greater than

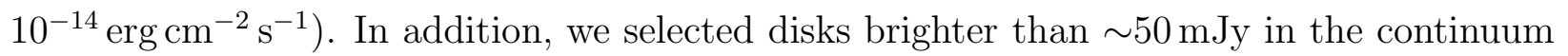
because target acquisition for fainter objects becomes challenging with VISIR. The 6 targets we selected for this campaign are TW Hya, CS Cha, VW Cha, T Cha, Sz 73, and Sz 102 (see Table 1 and 4 for their main properties). At the beginning of the second night we also observed the double-lined spectroscopic binary HD 34700A, which is the brightest infrared T Tauri couple in a quadruple system (Sterzik et al. 2005). Although HD 34700A did not have a published [Ne II] line detection, its near- and mid-infrared spectra present unusually strong PAH emission bands (e.g., Smith et al. 2004), possibly hinting to a high stellar far-UV flux (e.g., Geers et al. 2006), which may be indicative of a high EUV flux.

The systems we selected can be grouped into three categories based on their broad-band spectral energy distribution (SED). VW Cha, Sz 73, and Sz 102 have significant excess 
emission relative to the photospheric flux from near- through to far-infrared wavelengths (Gauvin \& Strom 1992; Hughes et al. 1994; Kessler-Silacci et al. 2006). Such broad SEDs can be well reproduced by radially continuous optically thick dust disks, extending from a few stellar radii out to hundreds of AU. TW Hya, CS Cha, and T Cha are classified as transition disks in the literature. Their SEDs present a strongly reduced (or lack of) near-infrared excess emission but large mid- and far-infrared emission. Detailed modeling of their SEDs points to relatively large inner dust cavities almost devoid of sub-micron- and micron-sized dust grains: 1-4 AU for TW Hya (Calvet et al. 2002; Ratzka et al. 2007), 43 AU for CS Cha (Espaillat et al. 2007), and 15 AU for T Cha (Brown et al. 2007). There is no evidence of gas inner holes in these systems: TW Hya is accreting disk gas at a rate of $\sim 5 \times 10^{-10} \mathrm{M}_{\odot} / \mathrm{yr}$ (Muzerolle et al. 2000), the spectroscopic binary CS Cha at $<10^{-8} \mathrm{M}_{\odot} /$ yr (Espaillat et al. 2007)1, while the small $(<10 \AA)$ but variable $\mathrm{H} \alpha$ equivalent width from T Cha suggests low-level and possibly episodic accretion (Alcala et al. 1993). Finally, the SED of HD 34700A has little excess emission at all wavelengths produced by a tenuous dust disk, possibly a debris disk (Sylvester \& Skinner 1996).

\subsection{Observations}

We performed long-slit high-resolution spectroscopy with the spectrograph VISIR mounted on the VLT telescope Melipal (Lagage et al.|2004). The observations were executed on 20, 21, and 22 February 2008. VISIR has a $256 \times 256$ BIB detector array with an image scale of 0 '.127/pixel. We acquired all targets with the PAH2-NEII filter. We used a slit width of $0^{\prime \prime} .4$ centered at $12.81 \mu \mathrm{m}$ to detect and spectrally resolve the $[\mathrm{Ne} \mathrm{II}]\left({ }^{2} P_{3 / 2}-{ }^{2} P_{1 / 2}\right)$

\footnotetext{
${ }^{1}$ the estimate from Espaillat et al. (2007) should be considered only an upper limit because the UV excess emission was computed assuming that CS Cha is a single star
} 
fine-structure line at $12.81355 \mu \mathrm{m}$ (Yamada et al. 1985). With this configuration we covered the spectral region between $\sim 12.79$ and $12.83 \mu \mathrm{m}$ with a resolution of $R \sim 30,000$ as measured from first light data (Käufl 2006) and from the FWHM of 5 narrow $\mathrm{O}_{3}$ sky lines from our spectra. This resolution corresponds to 3 VISIR pixels or $\sim 10 \mathrm{~km} / \mathrm{s}$ in velocity scale.

We applied the standard chopping/nodding technique to suppress the mid-infrared background. For each target the slit was positioned in the default North-South orientation and chopping/nodding was done along the slit with a throw of $8^{\prime \prime}$. Total on-source exposure times per science target were $1 \mathrm{~h}$ or longer. To correct the spectra for telluric absorption and to obtain an absolute flux calibration, we observed standard stars immediately before or after the science target and at a similar airmass. A summary of the observations is presented in Table 2 ,

\subsection{Data Reduction}

VISIR high-resolution spectra. For each nod position VISIR raw data are organized in cubes with the third axis having $2 n+1$ planes, where $n$ is the number of chopping cycles. For each chopping cycle $(i)$ two so-called Half-Cycle exposures are made: the $A_{i}$ image from the on-source position of the chopper, and the $B_{i}$ image from the off-source position of the chopper. The data cube stores the $A_{i}$ images in the odd planes and the average of the current and all previous $A_{i}-B_{i}$ images in the even planes. The last plane of the cube contains the average of all $A_{i}-B_{i}$ images and it is thus identical to the $2 \times n$ plane.

We process the raw data cubes with the VISIR pipeline version 3.2.1 (Lundin et al. 2008). First, 2D frames in each nod position are created by averaging all the Half-Cycle 
difference images in the data cube and low-level vertical stripes are removed. This step results in a set of images with a positive and a negative beam because the chop throw we chose is smaller than half of the VISIR field-of-view. Then, nodded images (images corrected for the telescope nodding) are produced by averaging the images in the two nod positions ( $\mathrm{A}$ and $\mathrm{B}$ ) and dividing them by 2 times the detector integration time. At this point each nodded image contains a double positive beam in the center and a negative beam on each side separated by the nodding(=chopping) throw. Pixels detected as bad in the Half-Cycle frames are now cleaned by interpolation with neighboring pixels. In parallel, a reference frame of the infrared background, not corrected for chopping or nodding, is also created from the first Half-Cycle of the first nod position. The nodded images as well as the infrared background image are corrected for the optical distortion, which is known analytically, and are shifted and added to form the final combined image using the offsets stored in the FITS header (the nodding cycle sequence used for VISR is AnBnBnAn). The last step before extraction is to fold the two negative beams into the central positive beam.

The spectrum is then extracted following the optimal extraction method by Horne (1986). In brief, the image is collapsed in the spectral dispersion direction to create a source profile, which is normalized and then expanded in spectral direction to obtain a weight map. The science frame is multiplied by the weight map and the spectrum is obtained by summing in spatial direction. This extraction method works well for sources where, in addition to narrow emission line(s), the continuum emission is also detected. For sources like CS Cha, where only a narrow emission line is detected (but not the continuum, see Fig. 1), the optimal extraction method fails. For this source and its calibrators we have performed the standard full aperture extraction (same weights within a defined spatial range of pixels). We tested that an aperture of 10 pixels gives the best $\mathrm{S} / \mathrm{N}$ in the extracted spectra. 
The wavelength calibration is done using the collapsed background frame in spatial direction and cross-correlating it to a synthetic model spectrum of the atmosphere. The dispersion relation is well approximated by a first order polynomial and the offset in pixels that maximizes the cross-correlation can be determined to an accuracy of 0.01 pixel $(\sim 0.03 \mathrm{~km} / \mathrm{s})$. We also compared the observed peak position of two photospheric absorption lines in the spectra of the K5 III star HD 136422 and the K4.5 III star HD 139127 to those from the MARCS model atmosphere of a K5 III star (HR 6705, the spectrum was kindly provided by L. Decin). This exercise demonstrates that the peak centroids can be determined to a lower accuracy, varying from a tenth up to a few $\mathrm{km} / \mathrm{s}$ (see also Table 3).

All steps described above have been applied to both the target and its standard star(s). In addition, the extracted spectrum of the standard star was flux calibrated using the model flux from the VISIR standard star catalog. From this calibration we created a spectral response function (Jy/ADU) that we applied at the wavelengths of the science spectrum. This step removes most of the "fringing" present in the VISIR spectra (the flux modulation with wavelengths has a peak to peak amplitude of about $15 \%$ and is found to be stable over periods of at least several months, van Boekel et al. 2009).

Spitzer/IRS spectra. As mentioned in Sect. 2.1, all targets except HD 34700 have published low- $(\mathrm{R} \sim 105)$ or high-resolution ( $\mathrm{R} \sim 600)$ IRS spectra with bright [Ne II] emission lines (see Table 1). We have reduced the archival IRS low-resolution spectrum of HD 34700 and report no detection of the [Ne II] line. In addition, we have reduced archival high-resolution IRS spectra for TW Hya and CS Cha obtained at different epochs than the already published spectra by Ratzka et al. (2007) and Espaillat et al. (2007). These spectra were acquired as part of the Spitzer GO program 30300 (PI, J. Najita) and are presented here to aid the interpretation of the VISIR results (Sect. 4). The data reduction of the IRS high- and low-resolution spectra followed the procedure outlined in Pascucci et al. (2006, 
2007, 2008) and Bouwman et al. (2008). The [Ne II] line fluxes from these new spectra are also reported in Table 1 (first entries in column 5).

\section{Results}

We detect and spectrally resolve [Ne II] emission lines from 4 out of 7 targets, specifically TW Hya, CS Cha, T Cha, and Sz 73. The [Ne II] emission detected with VISIR is found to be comparable to the angular resolution $\left(\sim 0.5^{\prime \prime}\right)$ estimated from both the mid-infrared continuum of our targets and from the telluric standards observed before or after the targets. The profiles of the lines are consistent with a single Gaussian profile (see Fig. 2). Table 3 provides the peak centroids (in the stellocentric frame), FWHMs, and fluxes of the [Ne II] lines computed assuming a Gaussian profile and a first-order polynomial for the continuum. In the case of non-detections (see Fig. 3), we fit a first-order polynomial within $\pm 100 \mathrm{~km} / \mathrm{s}$ of the star velocity. Table 3 provides the $3 \sigma$ upper limits to the [Ne II] flux computed from the RMS in the baseline-subtracted spectrum and assuming a line width of $10 \mathrm{~km} / \mathrm{s}$, equal to the instrument resolution (Sect. 2.2). Upper limits for broader lines simply scale by (FWHM/10). Figs. 2 and 3 illustrate our best fits to the detected lines and the hypothetical $3 \sigma$ upper limits when no line is detected.

The second-epoch Spitzer spectra of TW Hya and CS Cha presented here demonstrate that [Ne II] line fluxes are not constant in time and that the infrared continuum emission is also variable. More details on the line and continuum variability of TW Hya will be presented in an upcoming paper (Najita et al in prep.). The important result for the interpretation of these VISIR spectra is that changes of $\sim 30 \%$ in the line and/or in the continuum are possible even among the class of transition disks.

We do not detect the [Ne II] emission line in the Spitzer low-resolution spectrum of 
HD 34700A. This star is surrounded by a tenuous dust disk and is thus more similar to the optically thin dust disks studied by Pascucci et al. (2006) rather than to the protoplanetary disks presented in this paper. The [Ne II] non-detection suggests that very little or no primordial gas is left in the disk of HD 34700A (Pascucci et al. 2007), corroborating its identification as a debris disk.

In the following, we discuss in more detail the 6 protoplanetary disks with spectrally unresolved [Ne II] lines in their Spitzer spectra. We separate transition objects from radially continuous dust disks because their VISIR spectra appear remarkably different.

\section{4. [Ne II] emission from the disk of transition objects}

For the three transition disks TW Hya, CS Cha, and T Cha we detect and spectrally resolve [Ne II] lines located near the stellar velocity. In the case of CS Cha we detect only the emission line but not the continuum 2. For all sources [Ne II] line fluxes from VISIR spectra are lower than those measured from Spitzer spectra of factors between $\sim 0.5-0.8$. One possibility is that the $[\mathrm{Ne} \mathrm{II}]$ emission is extended beyond the $0.4^{\prime \prime}$ slit adopted in these observations. Spatially extended [Ne II] emission can certainly arise in outflow sources, as demonstrated by van Boekel et al. (2009) and by our sample of optically thick dust disks (see Sect. 5). However, no jets have been reported toward TW Hya and T Cha (Azevedo et al. 2007; Alcala et al. 1993) and only a compact (8-10 mas) outflow in $\mathrm{H} \alpha$ has been detected toward CS Cha (Takami et al. 2003). In Sect. 4.1 we also show that jets/outflows are not likely to be the main source of [Ne II] emission in transition

\footnotetext{
${ }^{2}$ The flux calibrated spectrum shown in Fig. 2 is the mean of the flux calibrated spectra from the 3 datasets, errors at each wavelength are the standard deviations from the 3 spectra on a common wavelength scale
} 
objects. Another explanation for the Spitzer-VLT flux difference is line and/or continuum variability. At least for CS Cha and TW Hya we know that line/continuum variability measured from 2-epochs of Spitzer spectra is $\sim 30 \%$, similar to the [Ne II] flux differences from the VLT and Spitzer spectra. We should also keep in mind that at the distance of TW Hya and T Cha (Table 4) our slit just covers out to $\sim 10$ AU from the central stars. As discussed later even [Ne II] emission from the disk surface can extend beyond several tens of AU. Further observations with wider slits and different orientation angles are necessary to constrain the spatial extension of the [Ne II] emission.

The most important result from the VISIR spectra is that the measured [Ne II] lines of transition disks are relatively narrow (ranging from $\sim 14 \mathrm{~km} / \mathrm{s}$ to $\sim 40 \mathrm{~km} / \mathrm{s}$ ) and peaked near the stellar velocity (see Table 3). These line characteristics are consistent with a disk origin for the emission 3 . We note that TW Hya, whose disk is almost seen face-on $\left(4 \pm 1^{\circ}\right.$, Pontoppidan et al. 2008), has the narrower line width. The inclinations of the disks around CS Cha and T Cha are not well constrained but likely closer to edge-on. Espaillat et al. (2007) successfully model the SED of CS Cha with a disk viewed at an inclination of $60^{\circ}$. T Cha has a high projectional velocity suggesting that it is viewed almost edge-on (Alcala et al. 1993). The [Ne II] line widths we measure seem to correlate with the disk viewing angle: the narrowest [Ne II] line is measured from the almost face-on disk of TW Hya, a broader line from the inclined disk of CS Cha, and the broadest line from the almost edge-on disk of $\mathrm{T}$ Cha. This trend also suggests that the [Ne II] line profiles are dominated by Keplerian rotation rather than by turbulence.

Further constraints on the region traced by the [Ne II] emission can come from modeling line profiles. In a recent study, Alexander (2008) showed that the velocity structure of a

\footnotetext{
${ }^{3}$ The line width from TW Hya reported by Herczeg et al. (2007) is about 1.6 times larger than our. Their spectrum has a poor $\mathrm{S} / \mathrm{N}$ and was obtained at an airmass as large as 1.8.
} 
photoevaporative disk wind results in a line profile that is different from that produced by a bound disk atmosphere. In particular, when a disk is viewed face-on the vertical component of the wind velocity leads to a profile that is about $\sim 10 \mathrm{~km} / \mathrm{s}$ in width (broader than the thermal line width) and blueshifted by $\sim 6-7 \mathrm{~km} / \mathrm{s}$. When a disk is viewed closer to edge-on, line profiles become broader $(\sim 30-40 \mathrm{~km} / \mathrm{s})$, are double peaked, dominated by Keplerian rotation, and centered at the stellar velocity. Fig. 4 compares the observed [Ne II] line profiles with the line profiles predicted by the standard photoevaporative disk wind model of Font et al. (2004) and Alexander (2008), the one in which there is no hole in the gas disk. We have chosen this model because our transition objects are still accreting disk gas, suggesting that there is no hole in the gas disk even if the inner dust disk is largely depleted of small dust grains (Sect. 2). The standard photoevaporative disk wind model assumes that the disk atmosphere is heated by the stellar EUV photons at $10^{4} \mathrm{~K}$ and requires just 3 input parameters to compute the [Ne II] line profiles: the stellar mass, the disk inclination, and the stellar ionizing flux $(\Phi)$. The profiles shown in Fig. 4 are for the star/disk parameters summarized in Table 4 and assuming a stellar ionizing flux of $10^{41}$ photons/s. There is an almost perfect match between the observed and predicted [Ne II] profile from the disk of TW Hya. The almost face-on disk of TW Hya has a blueshifted [Ne II] line fully consistent with the emission originating in a photoevaporative wind moving toward the observer (Alexander 2008). A static disk atmosphere would result in a line emission centered at the stellar velocity, clearly inconsistent with our observations. Thus, we consider this result as the first strong evidence for a disk being photoevaporated by its central star.

The [Ne II] line profiles of CS Cha and T Cha are broadly consistent with predictions from the photoevaporative disk wind model (Fig. 4), but there are also a few discrepancies, especially for the spectroscopic binary CS Cha. For this binary the predicted [Ne II] line is broader than observed. This could result from a disk viewed at less than $45^{\circ}$ inclination, 
but the emission should be slightly more blue-shifted than observed.

In the simplest assumption that neon atoms are ionized only by stellar EUV photons, we can use the observed [Ne II] line luminosities to estimate the stellar ionizing flux $\Phi$ reaching the disk surface. The reason is that [Ne II] line fluxes scale approximately linearly with $\Phi$ (for $\Phi \leq 10^{43}$ photons/s) while the shape of the line profile is insensitive to the value of $\Phi$ (Alexander 2008). The comparison between the observed and predicted line luminosities yields stellar ionizing fluxes of $2.5,13$, and $1.9 \times 10^{41}$ photons/s for TW Hya, CS Cha, and T Cha respectively. These values have an uncertainty of a factor of 2-3 dominated by the uncertainty in the fraction of neon atoms that exist as $\mathrm{Ne}^{+}$in the disk surface. Alexander (2008) keeps this fraction constant to $1 / 3$ while more detailed gas models suggest that it could be as high as 1 (Hollenbach \& Gorti 2009). The ionizing rates we compute from the [Ne II] lines seem plausible for accreting T Tauri stars. For TW Hya far-UV observations suggest values of $7 \times 10^{39}$ or $5 \times 10^{41}$ depending on how much EUV emission from the accretion shock is attenuated by the accretion streams (Herczeg 2007). As a next step we can use the values of $\Phi$, in combination with star and disk masses, to estimate the disk wind rates and the disk lifetimes if EUV-driven photoevaporation would be the only disk dispersal mechanism. Using the results from Font et al. (2004) we find disk wind rates ranging from $\sim 2$ to $5 \times 10^{-10} \mathrm{M}_{\odot} / \mathrm{yr}$. At these rates only the relatively low-mass disk of $\mathrm{T}$ Cha $\left(0.003 \mathrm{M}_{\odot}\right.$, Lommen et al. 2008) could be photoevaporated in $\sim 10 \mathrm{Myr}$. The disks of TW Hya and CS Cha would disperse in several tens of millions of years. However, a more realistic estimate for the disk lifetime should include on-going mass loss onto the star via viscous accretion. In addition, disk lifetimes can be further reduced once the viscous accretion infall rate falls below the photoevaporation rate. At this point a gap opens in the gas disk and the direct EUV flux from the star is expected to disperse the entire disk in only $\sim 10^{5}$ yr. As we discuss in Sect. 6, at least two of our transition objects may be on the verge of opening an inner gap in the gas disk. 
Finally, it is worth commenting on the extension of the [Ne II] emission in the photoevaporative disk models. Calculations from Alexander (priv. comm.) show that more than $90 \%$ of the [Ne II] emission arises inside $\sim 2 \times 9\left(M_{\star} / M_{\odot}\right)$ AU. These radial distances are covered by our $0.4^{\prime \prime}$ slit for CS Cha, are comparable to half the slit width for TW Hya, and smaller by a factor of 2 for T Cha. This shows that we certainly missed some [Ne II] flux (especially from the disks of TW Hya and T Cha) even in the case that ionization is solely from EUV photons. Stellar X-rays could contribute to the gas ionization at larger disk radii (Glassgold et al. 2007) and the X-ray heated gas might also participate in the photoevaporative flow (Ercolano et al. 2009). If X-rays substantially contribute to the [Ne II] fluxes then the stellar ionizing fluxes we calculated above should be viewed only as upper limits. It would be extremely interesting in the future to compare the observed [Ne II] profiles to those predicted by photoevaporating X-ray-heated gas disks.

\subsection{Can jets explain the [Ne II] emission from transition disks?}

The observations of the T Tau complex from van Boekel et al. (2009) demonstrate that [Ne II] emission can also originate from shocks generated by protostellar outflows/jets. Because of the high ionization potential of neon atoms $(21.6 \mathrm{eV})$, substantial ionization can be only produced by fast shocks with speeds $\geq 70 \mathrm{~km} / \mathrm{s}$ for typical pre-shock densities of $10^{4} \mathrm{~cm}^{-3}$ (also called J shocks, Hollenbach \& McKee 1989). For this type of strong radiative shocks the shocked gas moves at almost the shock velocity. van Boekel et al. (2009) argue that these high velocities may be reached in the outflow from the T Tau S source which has a sight velocity of only $\sim 40 \mathrm{~km} / \mathrm{s}$, but it is likely to be in the plane of the sky. We now show that it is very unlikely that jets/outflows could explain the [Ne II] emission of transition disks. First, let us consider the case of TW Hya. Its disk is seen almost face-on so we would expect any jet/outflow to be almost in the direction of the observer and the sight 
velocity to be close to the actual shock velocity. The sight velocity of the [Ne II] emission from TW Hya is just $-6 \mathrm{~km} / \mathrm{s}$ and the line is relatively narrow (deconvolved FWHM of $\sim 10 \mathrm{~km} / \mathrm{s}$ ). If this velocity is representative for the shock velocity as predicted from $\mathrm{J}$ shock models, it would be unable to appreciably ionize neon atoms. Corroborating this assertion, Hollenbach \& Gorti (2009) show that the [Ne II] line luminosity produced by the postshock region of a radiative shock depends linearly on: $\dot{M}_{w} v_{s}^{2}$, where $\dot{M}_{w}$ is the protostellar mass wind loss rate and $v_{s}$ is the shock velocity. The wind mass loss rate scales with the stellar mass accretion rate as $\dot{M}_{w} \simeq 0.01-0.1 \times \dot{M}_{a c c}$ (Hartigan et al. 1995; White \& Hillenbrand 2004). Given the low stellar accretion rates of transition disks, the [Ne II] fluxes reported in Table 3 could be reached only with shock velocities ranging from several hundreds of $\mathrm{km} / \mathrm{s}$ to thousands of $\mathrm{km} / \mathrm{s}$, clearly inconsistent with the small velocity shifts and relatively small FWHM of the observed [Ne II] lines. Finally, line widths from shocked gas are expected to be quite broad (several tens of $\mathrm{km} / \mathrm{s}$ ) regardless of the observer viewing angle (e.g., Hartigan et al. 1987), thus shocked gas cannot reproduce the observed trend between the FWHM of [Ne II] lines and the disk inclination. Although shocks generated by jets/outflows are unlikely to explain the [Ne II] emission from transition disks, they may dominate the [Ne II] emission from less-evolved optically thick dust disks (see Guedel et al. 2009 and next Section).

\section{Jets/outflows dominate the [Ne II] emission of classical optically thick disks}

The three optically thick dust disks observed here have spectrally unresolved [Ne II] fluxes comparable to those of transition disks. Nevertheless, we do not detect any [Ne II] line close to the stellar velocity in their VISIR spectra. Only in the case of $\mathrm{Sz} 73$ we detect a broad $(\sim 60 \mathrm{~km} / \mathrm{s})$ emission line at $\sim-100 \mathrm{~km} / \mathrm{s}$ with respect to the stellar velocity and a line flux that is just $\sim 30 \%$ lower than the Spitzer [Ne II] flux. The large FWHM in 
combination with the large blueshift point to a jet/outflow origin for the [Ne II] emission. The case of $\mathrm{Sz} 73$ well illustrates that when jets/outflows are present the [Ne II] emission they produce can dominate over the disk emission. This is in line with the observations of the T Tau complex from van Boekel et al. (2009).

The [Ne II] non-detections of Sz 102 and VW Cha are also consistent with this scenario. If the [Ne II] emission measured with Spitzer would originate in a disk, only relatively broad lines $(\mathrm{FWHM}>50 \mathrm{~km} / \mathrm{s})$, compatible only with close to edge-on disks, would be undetected in our VISIR spectra. Interestingly, VW Cha and Sz 102 are known to have outflows at position angles (from N to E) of 90 and 99 degrees respectively (Bally et al. 2006; Krautter 1986; Graham \& Hever 1988). Since we did not change the default slit orientation of VISIR $\left(\mathrm{PA}=0^{\circ}\right)$ we acquired spectra in a direction perpendicular to the known outflows. Thus, our non-detections can be reconciled with the Spitzer observations if most of the [Ne II] emission arises in jets/outflows, which can be verified with future high-resolution spectra covering the region of the known outflows.

\section{On the onset of centrally-driven photoevaporation}

Viscous accretion is thought to be the dominant disk dispersal mechanism but alone cannot explain the relatively short $(\lesssim 10 \mathrm{Myr})$ disk dispersal timescales inferred from observations (e.g. review by Dullemond et al. 2007). High-energy stellar photons can heat and ionize the disk surface and induce photoevaporation (i.e. disk mass loss) from outer disk regions. Exactly when photoevaporation becomes an important disk dispersal mechanism and how much disk mass it can remove is still a matter of debate. FUV and X-ray photons can penetrate much larger column densities of gas than stellar EUV photons. As a consequence, they are expected to drive efficient photoevaporation from the early times of disk evolution at rates that may exceed the EUV photoevaporation rate by 
one or even two orders of magnitudes (Gorti \& Hollenbach 2009; Ercolano et al. 2009). Stellar EUV photons start to penetrate the disk wind, and thus reach the disk surface, only when accretion rates fall below $\sim 10^{-8} \mathrm{M}_{\odot} / \mathrm{yr}$, corresponding to hydrogen column density screens of $\mathrm{N}(\mathrm{H}) \lesssim 10^{17} \mathrm{~cm}^{-2}$ (Hollenbach \& Gorti 2009). Although EUV photons likely produce little mass loss at all stages (Gorti \& Hollenbach 2009), they can help to quickly $\left(\sim 10^{5} \mathrm{yr}\right)$ disperse remaining disk gas once stellar accretion rates fall below a few $10^{-10} \mathrm{M}_{\odot} / \mathrm{yr}$ (Alexander et al. 2006a).

Recently, Currie et al. (2009) have argued that the large number of evolved dust disks in the 5 Myr-old cluster NGC 2362 is inconsistent with predictions from the standard UV photoevaporation model, in which EUV-driven photoevaporation follows viscous accretion (Clarke et al. 2001; Alexander et al. 2006b). Indeed, these models predict a paucity of evolved disks because once the accretion inflow rates fall below the photoevaporation rate the disk dispersal is fast $\left(\sim 10^{5} \mathrm{yr}\right)$. However, model predictions are directly relevant only to the evolution of the gas disk component. In order to translate the gas evolution into dust evolution, Alexander et al. (2006b) made the simplistic assumption that the dust is coupled to the gas throughout the entire disk evolution. This is certainly unrealistic because grain growth, observed in almost all protoplanetary disks (e.g. Natta et al. 2007), results in dust settling, i.e. decoupling of the dust from the gas disk (Dullemond \& Dominik 2004). A more direct way to test photoevaporation models is to constrain the evolution of the gas disk through observations of gas lines tracing the accreting as well as the possibly photoevaporating material. In what follows we will take this approach to speculate on the onset of photoevaporation.

We start by discussing our sample of probably less evolved classical optically thick disks with radially continuous dust distribution. The $\mathrm{H} \alpha$ equivalent widths from these three systems are greater than $50 \AA$ (Hughes et al. 1994; Guenther et al. 2007), indicating 
that the central stars are accreting nebular gas. This is corroborated by the detection of outflows and large near-infrared excess emission, both characteristic to actively accreting disks (Hartigan et al. 1995). In these systems we do not find evidence for on-going disk photoevaporation. The [Ne II] line flux upper limits at the stellar velocity imply that not only EUV photons but also stellar X-rays are efficiently screened by the protostellar wind and accretion columns, requiring large gas column densities $\left(\mathrm{N}_{H} \gtrsim 10^{22} \mathrm{~cm}^{-2}\right.$, e.g. Guedel et al. 2007). If column densities exceed $\sim 10^{24} \mathrm{~cm}^{-2}, \mathrm{FUV}$ photons are also significantly attenuated (Hollenbach \& Gorti 2009). For lower column densities, photoevaporation driven by FUV photons could be occurring and we would not detect it because FUV photons have energies lower than the ionization potential of neon atoms $(21.6 \mathrm{eV})$. These systems may be at the same evolutionary stage as the T Tau complex.

Recently, Najita et al. (2009) reported spectrally resolved [Ne II] lines from two disks in the Taurus-Auriga star-forming region: AA Tau, a classical optically thick disk whose star presents periodic accretion rate bursts (Bouvier et al. 2007); and GM Aur, a transition disk whose central star is accreting at a level comparable to that of classical T Tauri stars $\left(10^{-8} \mathrm{M}_{\odot} / \mathrm{yr}\right.$, Gullbring et al. 1998). The [Ne II] lines are found to be centered at the stellar velocity suggesting that the emission originates from a static disk atmosphere very likely heated by stellar X-rays. However, the measured fluxes are factors of 2 and 3 lower than the spectrally unresolved fluxes from Spitzer spectra. One possible explanation is that most of the undetected [Ne II] emission originates far from the central stars, in ambient gas shocked by a jet/outflow. This seems very plausible for AA Tau, whose optical spectrum has forbidden emission lines typical of high-velocity jets (e.g. [NII] $6530 \AA$, Hirth et al. 1997) and a jet has also been imaged with HST/STIS and Goddard Fabry Perot spectrograph (Cox et al. 2005). The [Ne II] emission reported by Najita et al. (2009) does not seem to trace photoevaporating gas in these two systems, possibly because X-ray heated gas producing $[\mathrm{Ne}$ II] emission is slightly cooler $(\sim 5,000 \mathrm{~K})$ and thus more bound than the 
EUV-heated gas.

Finally, the transition disks presented here have [Ne II] line profiles consistent with emission from a photoevaporative disk wind indicating on-going photoevaporation at this evolutionary stage. In the case of TW Hya, the stellar accretion rate is close to the limit where EUV-driven photoevaporation could start opening a gap in the gas disk. The small but varying $\mathrm{H} \alpha$ equivalent width of $\mathrm{T}$ Cha suggests that this transition object may also be on the verge of clearing a gas hole. Interestingly, TW Hya and T Cha are also thought to be relatively old, older than 5 Myr (Webb et al. 1999; van den Ancker et al. 1998, see also Table 1). CS Cha is the youngest among our transition disks. Its upper limit to the stellar accretion rate is close to what is needed for EUV photons to start penetrating the disk wind, placing it at an earlier evolutionary stage than TW Hya and T Cha (though the evolution of the inner disk is certainly affected by presence of the stellar companion).

In overall, these results support the evolutionary picture in which EUV-driven photoevaporation occurs at later times in the disk evolution and the mass loss rates can be only modest. We should also note that if stellar X-rays substantially contribute to the $[\mathrm{Ne}$ II] emission of transition disks then the stellar ionizing flux $\Phi$ calculated in Sect. 4 would be only upper limits, further reducing the efficiency of EUV-driven photoevaporation. Future observations should aim at expanding the sample of classical and transition disks with spectrally resolved [Ne II] lines and accretion rate measurements. In addition, it would be extremely valuable to compute [Ne II] line profiles from X-ray heated photoevaporating gas that could be directly compared to observations. 


\section{Summary}

We used the high-resolution spectrograph VISIR on the VLT to spectrally resolve [Ne II] emission lines previously detected with the Spitzer Space Telescope in a sample of young protoplanetary disks. Our main results can be summarized as follows:

- We detect and spectrally resolve the [Ne II] lines from the 3 transition disks in our sample. The line centroids, widths, and intensities indicate that the [Ne II] emission arises in a disk. Models of disk wind photoevaporation well reproduce the observed line profiles strongly suggesting on-going photoevaporation driven by stellar EUV photons at this evolutionary stage.

- From the sample of optically thick radially continuous dust disks we only detect a strong and broad [Ne II] emission blueshifted by $-100 \mathrm{~km} / \mathrm{s}$ toward $\mathrm{Sz} 73$. This points to a jet/outflow origin for the [Ne II] emission. The [Ne II] non-detection in the other two systems with known jets also suggest that most of the spectrally unresolved [Ne II] emission detected with Spitzer does not arise from the disk but rather from surrounding gas shocked by jets/outflows.

These results provide the first observational evidence for centrally-driven disk photoevaporation. Because transition disks have already undertaken significant evolution in comparison to the radially continuous dust disks, our results demonstrate that EUV-driven photoevaporation can occur only at a late stage in the disk evolution.

We thank R. D. Alexander for proving the [Ne II] line profiles from photoevaporative disk winds and for extremely useful discussions. We would also like to thank F. Lahuis for making available the high-resolution Spitzer/IRS spectra of $\mathrm{Sz} 73, \mathrm{Sz} 102$, T Cha, and VW Cha and L. Decin for the MARCS model atmosphere of HR 6705. We are also grateful 
to D. Hollenbach, U. Gorti, B. Ercolano, J. Najita, J. Carr, and D. Apai for valuable discussions, and an anonymous referee for helpful suggestions. IP is pleased to acknowledge support through NASA contract 90035375.

Facilities: VLT (VISIR), Spitzer (IRS). 


\section{REFERENCES}

Alcala, J. M., Covino, E., Franchini, M., Krautter, J., Terranegra, L., Wichmann, R. 1993, A\&A, 272, 225

Alexander, R. D., Clarke, C. J., Pringle, J. E. 2006a, MNRAS, 369, 216

Alexander, R. D., Clarke, C. J., Pringle, J. E. 2006b, MNRAS, 369, 229

Alexander, R. D. 2008, MNRAS, L391, 64

Azevedo, R., Folha, D. F. M., Gameiro, J. F., Calvet, N. 2007, ApJ, 670, 1234

Bally, J., Walawender, J., Luhman, K. L., Fazio, G. 2006, AJ, 132, 1923

Barrado Y Navascués, D. 2006, A\&A, 459, 511

Bouvier, J., Alencar, S. H. P., Boutelier, T. 2007, A\&A, 463, 1017

Bouwman, J. Henning, Th., Hillenbrand, L. A., Meyer, M. R., Pascucci, I., Carpenter, J., Hines, D., Kim, J. S., Silverstone, M. D., Hollenbach, D., Wolf, S. 2008, ApJ, 683, 479

Brandner, W., Alcala, J. M., Kunkel, M., Moneti, A., Zinnecker, H. 1996, A\&A, 307, 121

Brown, J. M., Blake, G. A., Dullemond, C. P. 2007, ApJ, L664, 107

Calvet, N., Hartmann, L., Strom, S. E. 2000, in Protostars and Planets IV (Book - Tucson: University of Arizona Press; eds Mannings, V., Boss, A.P., Russell, S. S.), p. 377

Calvet, N., D'Alessio, P., Hartmann, L., Wilner, D., Walsh, A., Sitko, M. 2002, ApJ, 568, 1008

Clarke, C. J., Gendrin, A., Sotomayor, M. 2001, MNRAS, 328, 485 
Cox, A. W., Hilton, G. M., Williger, G. M., Grady, C. A., Woodgate, B. 2005, AAS, 207, 7419

Currie, T., Kenyon, S. J., Balog, Z., Rieke, G., Bragg, A., Bromley, B. 2008, ApJ, 672, 558

Currie, T., Lada, C. J., Plavchan, P., Robitaille, T. P., Irwin, J., Kenyon, S. J. 2009, ApJ, 698,1

Cutri, R. M., Skrutskie, M. F., van Dyk, S. et al. 2003, 2MASS All Sky Catalog of point sources

Dullemond, C. P. \& Dominik, C. 2004, A\&A, 421, 1075

Dullemond, C. P., Hollenbach, D., Kamp, I., D'Alessio, P. 2007, in Protostars and Planets V, B. Reipurth, D. Jewitt, and K. Keil (eds.), University of Arizona Press, Tucson, 951 pp., 2007., p.555-572

Ercolano, B., Drake, J. J., Raymond, J. C., Clarke, C. C. 2008, ApJ, 688, 398

Ercolano, B., Clarke, C. J., \& Drake, J. J. 2009, ApJ, 699, 1639

Espaillat, C., Calvet, N., D’Alessio, P. et al. 2007, ApJ, L664, 111

Flaccomio, E., Stelzer, B., Sciortino, S., Micela, G., Pillitteri, I., Testi, L. 2009, A\&A in press (arXiv:0906.4700)

Font, A. S., McCarthy, I. G., Johnstone, D., Ballantyne, D. R. 2004, ApJ, 607, 890

Gauvin, L. S. \& Strom, K. M. 1992, ApJ, 385, 217

Geers, V. C., Augereau, J.-C., Pontoppidan, K. M., Dullemond, C. P., Visser, R., Kessler-Silacci, J. E., Evans, N. J., II, van Dishoeck, E. F., Blake, G. A., Boogert, A. C. A., Brown, J. M., Lahuis, F., Merín, B. 2006, A\&A, 459, 545 
Glassgold, A. E., Najita, J. R., Igea, J. 2007, ApJ, 656, 515

Gorti, U. \& Hollenbach, D. 2008, ApJ, 683, 287

Gorti, U. \& Hollenbach, D. 2009, ApJ, 690, 1539

Graham, J. A. \& Heyer, M. H. 1988, PASP, 100, 1529

Guedel, M., Skinner, S. L., Mel'Nikov, S. Yu., Audard, M., Telleschi, A., Briggs, K. R. 2007, A\&A, 468, 529

Guedel, M., van Boekel, R., Lahuis, F., Najita, J., Henning, T., Glassgold, A., van Dishoeck, E., Carr, J., Briggs, K. 2009 (http://www.ipac.caltech.edu/spitzer2008/talks/ManuelGuedel.html)

Guenther, E. W., Esposito, M., Mundt, R., Covino, E., Alcalá, J. M., Cusano, F., Stecklum, B. 2007, A\&A, 467, 1147

Gullbring, E., Hartmann, L., Briceno, C., Calvet, N. 1998, ApJ, 492, 323

Hartigan, P., Raymond, J., Hartmann, L. 1987, ApJ, 316, 323

Hartigan, P., Edwards, S., Ghandour, L. 1995, ApJ, 452, 736

Herczeg, G. J., Najita, J. R., Hillenbrand, L. A., Pascucci, I. 2007, ApJ, 670, 509

Herczeg, G. J. 2007, IAUS, 243, 147

Hernández, J., Hartmann, L., Megeath, T., Gutermuth, R., Muzerolle, J., Calvet, N., Vivas, A. K., Briceño, C., Allen, L., Stauffer, J., Young, E., Fazio, G. 2007, ApJ, 662, 1067

Hirth, G. A., Mundt, R., Solf, J. 1997, A\&AS, 126, 437 
Hollenbach, D., Gorti, U., Meyer, M., Kim, J. S., Morris, P., Najita, J., Pascucci, I., Carpenter, J., Rodmann, J., Brooke, T., Hillenbrand, L., Mamajek, E., Padgett, D., Soderblom, D., Wolf, S., Lunine, J. 2005, ApJ, 631, 1180

Hollenbach, D. \& McKee, C. F. 1989, ApJ, 342, 306

Hollenbach, D. \& Gorti, 2009, ApJ, submitted

Horne, K. 1986, PASP, 98, 609

Hughes, J., Hartigan, P., Krautter, J., Kelemen, J. 1994, AJ, 108, 1071

Joergens, V. \& Guenther, E. 2001, A\&A, L379, 9

Käufl, H.-U. 2006, in Planetary nebulae beyond the Milky Way, Proceedings of the ESO workshop held in Garching, Germany, 19-21 May 2004

Kessler-Silacci, J., Augereau, J.-C., Dullemond, C. P. 2006, ApJ, 639, 275

Krautter, J.1986, A\&A, 161, 195K

Lagage, P. O., Pel, J. W., Authier, M. et al. 2004, The Messenger 117, 12

Lahuis, F., van Dishoeck, E. F., Blake, G. A., Evans, N. J., II, Kessler-Silacci, J. E., Pontoppidan, Klaus M. 2007, ApJ, 665, 492

Lawson, W. A., Lyo, A., \& Muzerolle, J. 2004, MNRAS, 351, L39

Lecavelier des Etangs, A., Vidal-Madjar, A., Roberge, A., Feldman, P. D., Deleuil, M., André, M., Blair, W. P., Bouret, J.-C., Désert, J.-M., Ferlet, R., Friedman, S., Hébrard, G., Lemoine, M., Moos, H. W. 2001, Nature, 412, 706

Lommen, D., Jorgensen, J. K., van Dishoeck, E. F., Crapsi, A. 2008, A\&A, 481, 141

Luhman, K. L. to appear in the Handbook of Star Forming Regions, ASP Monograph Series 
Lundin, L. K. 2008, VLT VISIR Pipeline User Manual

Mamajek, E. E. 2005, ApJ, 634, 1385

Meijerink, R., Glassgold, A. E., Najita, J. R. 2008, ApJ, 676, 518

Melo, C. H. F. 2003, A\&A, 410, 269

Muzerolle, J., Calvet, N., Hartmann, L. 1998, ApJ, 492, 743

Muzerolle, J., Hillenbrand, L., Calvet, N., Hartmann, L., \& Briceño, C. 2000, ApJ, 545, L141

Najita, J. R., Doppmann, G. W., Bitner, M. A. et al. 2009, ApJ, 697, 957

Natta, A., Testi, L., Calvet, N., Henning, Th., Waters, R., Wilner, D. 2007, 767, in Protostars and Planets V, B. Reipurth, D. Jewitt, and K. Keil (eds.), University of Arizona Press, Tucson

Pascucci, I., Gorti, U., Hollenbach, D., Najita, J., Meyer, M. R., Carpenter, J. M., Hillenbrand, L. A., Herczeg, G. J., Padgett, D. L., Mamajek, E. E., Silverstone, M. D., Schlingman, W. M., Kim, J. S., Stobie, E. B., Bouwman, J., Wolf, S., Rodmann, J., Hines, D. C., Lunine, J., Malhotra, R. 2006, ApJ, 651, 1177

Pascucci, I., Hollenbach, D., Najita, J., Muzerolle, J., Gorti, U., Herczeg, G. J., Hillenbrand, L. A., Kim, J. S., Carpenter, J. M., Meyer, M. R., Mamajek, E. E., Bouwman, J. 2007, ApJ, 663, 383

Pascucci, I., Apai, D., Hardegree-Ullman, E. E., Kim, J. S., Meyer, M. R., Bouwman, J. 2008, ApJ, 673, 477

Pascucci \& Tachibana 2009, in "Protoplanetary Dust", Cambridge univ. Press 
Pontoppidan, K. M., Blake, G. A., van Dishoeck, E. F., Smette, A., Ireland, M. J., Brown, J. 2008, ApJ, 684, 1323

Ratzka, Th., Leinert, Ch., Henning, Th., Bouwman, J., Dullemond, C. P., Jaffe, W. 2007, A\&A, 471, 173

Roberge, A., Weinberger, A. J., Redfield, S., Feldman, P. D. 2005, ApJ, L626, 105

Sylvester, R. J. \& Skinner, C. J. 1996, MNRAS, 283, 457

Smith, T. L., Clayton, G. C., Valencic, L. 2004, AJ, 128, 357

Sterzik, M. F., Melo, C. H. F., Tokovinin, A. A., van der Bliek, N. 2005, A\&A, 434, 671

Takami, M., Bailey, J., Chrysostomou, A. 2003, A\&A, 397, 675

van Boekel, R., Guedel, M., Henning, Th., Lahuis, F., Pantin, E. A\&A in press

van den Ancker, M. E., de Winter, D., Tjin A Djie, H. R. E. 1998, A\&A, 330, 145

Webb, R. A., Zuckerman, B., Platais, I., Patience, J., White, R. J., Schwartz, M. J., McCarthy, C. 1999, ApJ, L512, 63

Weintraub, D. A., Kastner, J. H., Bary, J. S. 2000, ApJ, 541, 767

White, R. J. \& Hillenbrand, L. A. 2004, ApJ, 616, 998

Yamada, C., Kanamori, H., Hirota, E. 1985, J. Chem. Phys., 83, 552 


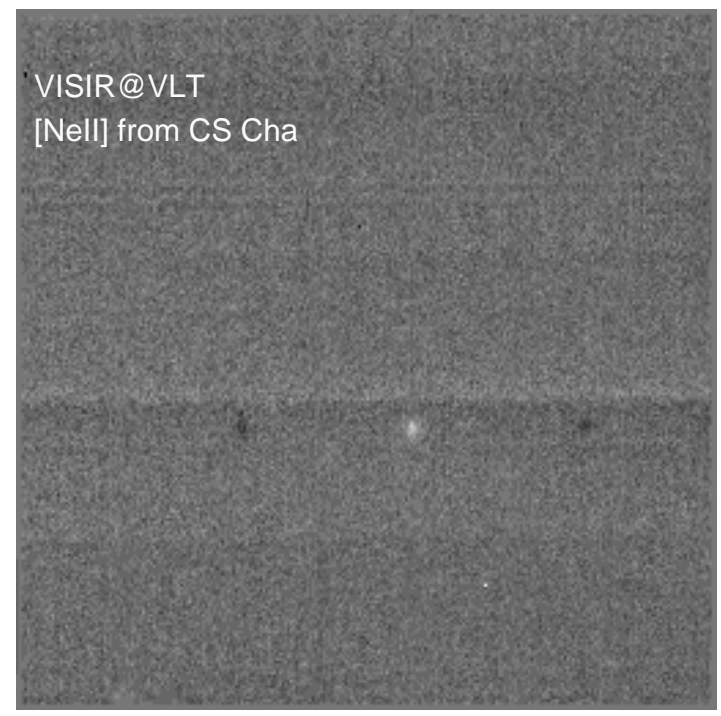

Fig. 1.- Combined image of CS Cha from the first dataset (February 22). The [Ne II] emission is clearly detected both in the positive and in the negative beams but there is no detection of the continuum emission. The combined images from the other two datasets are similar and therefore not shown here. 


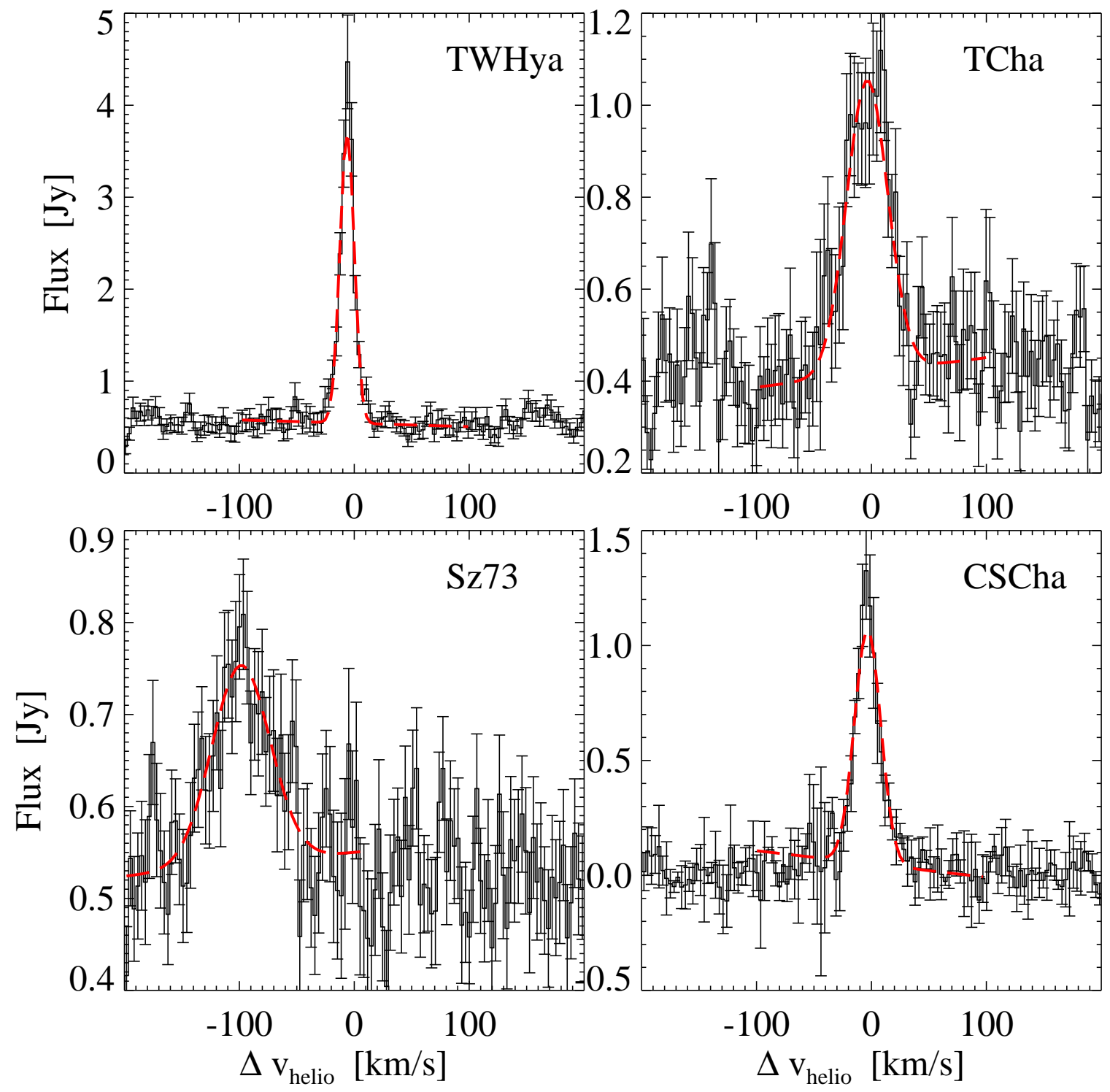

Fig. 2.- Sources for which we detect [Ne II] emission lines in their VISIR spectra. The $\mathrm{x}$-axis gives the velocity in the stellocentric frame (see Table 1 for the stellar heliocentric radial velocity). On top of the continuum emission we overplot the best Gaussian fits to the data (dashed lines). In the case of CS Cha the continuum emission is not detected (see also Fig. (1). 

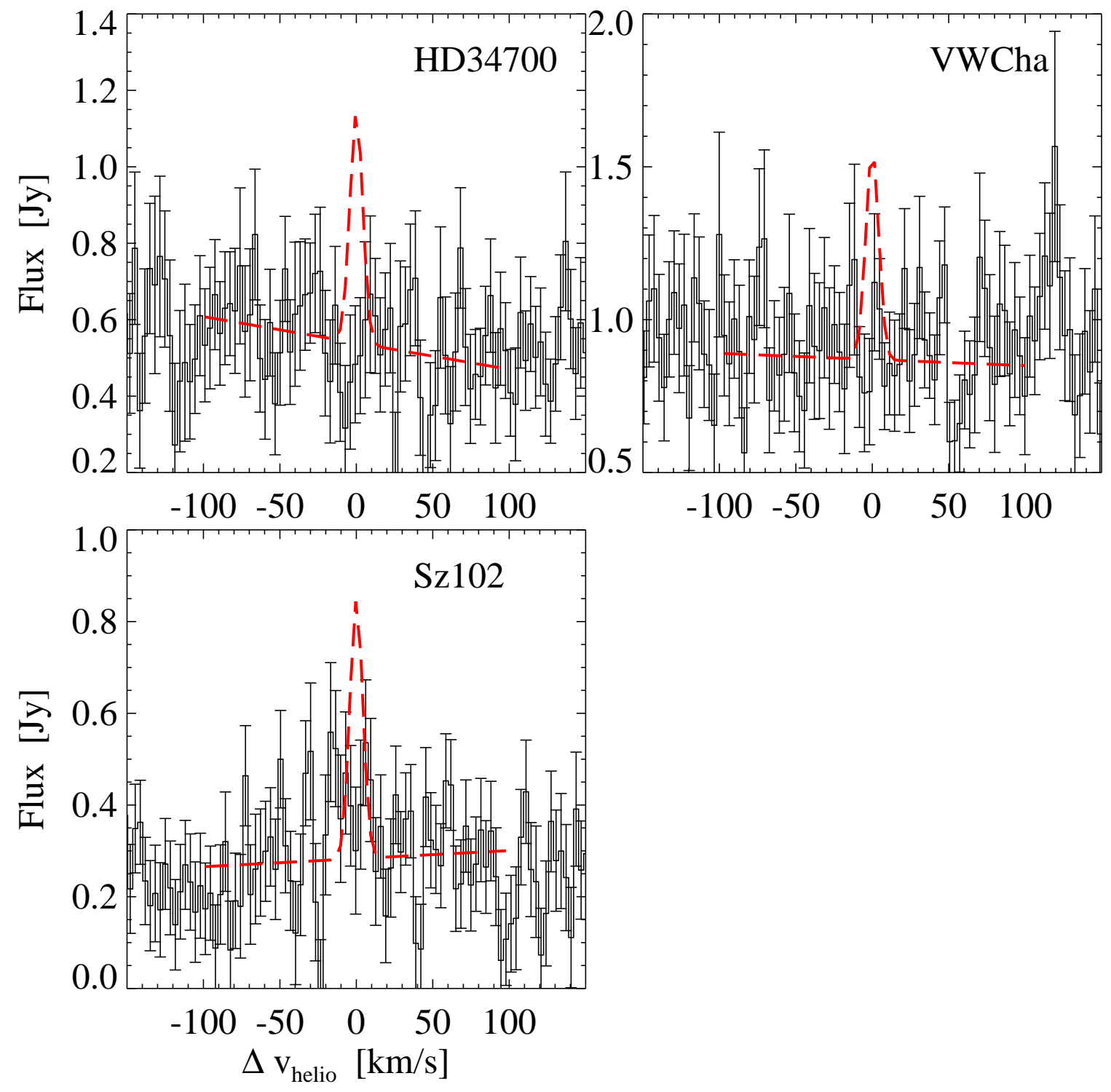

Fig. 3.- Sources for which we do not detect any [Ne II] emission line in their VISIR spectra. The $\mathrm{x}$-axis gives the velocity in the stellocentric frame. On top of the continuum emission we overplot the hypothetical $3 \sigma$ upper limits (dashed lines) reported in Table 3 for line widths equal to $10 \mathrm{~km} / \mathrm{s}$. Note that spectrally unresolved [Ne II] lines have been detected with Spitzer toward VW Cha and Sz 102 (Table 1). Our non-detections suggest that most of the [Ne II] emission measured with the Spitzer Space Telescope is not originating in a disk but likely in a jet/ouflow. 


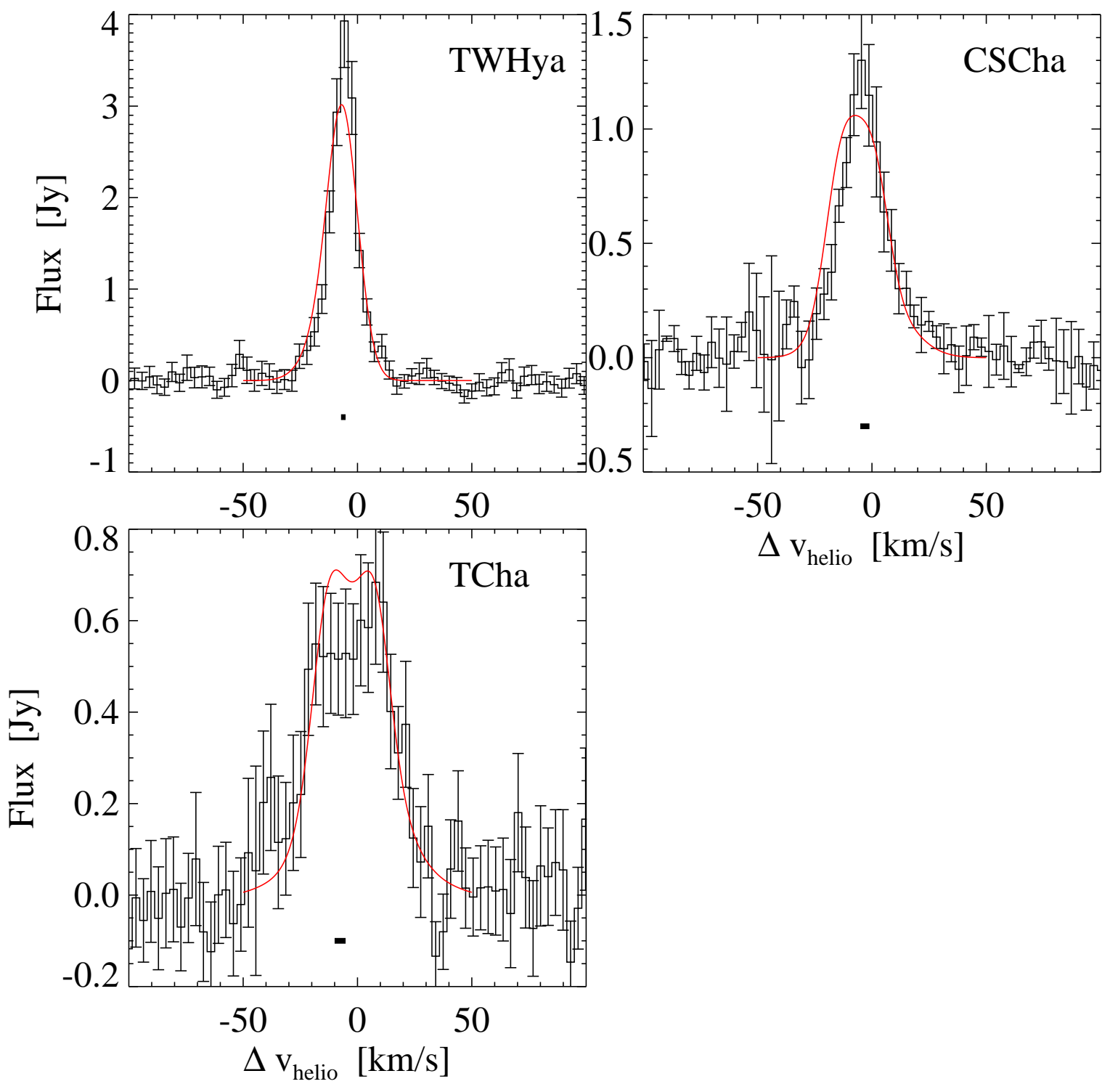

Fig. 4.- Comparison between observed and predicted (red solid line) line profiles for the group of transition disks (velocity in the stellocentric frame). Predicted line profiles are for the standard photoevaporative disk wind assuming an ionizing flux $\Phi$ of $10^{41}$ photons/s and the stellar mass and disk inclination in Table 4. Models have been degraded to the spectral resolution of VISIR and scaled by the factors reported in the last column of Table 4 to match the observed and predicted [Ne II] line luminosities. The black thick line below the [Ne II] emission shows the total uncertainty in the observed [Ne II] peak position. Note the excellent match between the observed and predicted line profile from the almost face-on disk of TW Hya suggesting that the [Ne II] emission originates from a photoevaporative disk 
Table 1. Stellar properties and [Ne II] fluxes measured with the Spitzer Space Telescope

\begin{tabular}{|c|c|c|c|c|c|c|}
\hline Source & 2MASS Ja & $\begin{array}{c}\mathrm{v}_{\mathrm{rad}}{ }^{\mathrm{b}} \\
(\mathrm{km} / \mathrm{s})\end{array}$ & $\begin{array}{l}\text { Age } \\
(\mathrm{Myr})\end{array}$ & Ref. & $\begin{array}{c}{[\mathrm{NeII}] \text { flux }} \\
\left(\times 10^{-14} \mathrm{erg} \mathrm{cm}^{-2} \mathrm{~s}^{-1}\right)\end{array}$ & Ref. \\
\hline HD $34700 \mathrm{Aa}^{*}$ & $05194387+0539406$ & $68.0 \pm 0.2$ & $1-10$ & 1 & - & 11 \\
\hline TW Hya & $11015191-3442170$ & $12.2 \pm 0.5$ & $\sim 10$ & 2,3 & $4.5 \pm 0.3,5.7$ & 11,12 \\
\hline CS Cha* & $11022491-7733357$ & $14.9 \pm 2$ & $\sim 2$ & $4,5,6$ & $3.4 \pm 0.1,4.3$ & 11,13 \\
\hline VW Cha* & $11080148-7742288$ & $17.2 \pm 0.5$ & $\sim 2$ & 5,6 & $2.8 \pm 0.4$ & 14 \\
\hline T Cha & $11571348-7921313$ & $14.0 \pm 1.3$ & $>12$ & 5,7 & $3.2 \pm 0.2$ & 14 \\
\hline $\mathrm{Sz} 73$ & $15475693-3514346$ & $-3.3 \pm 2.5$ & $2.6-5.4$ & 8,9 & $1.6 \pm 0.2$ & 14 \\
\hline Sz 102 & $16082972-3903110$ & 5 & $\ldots$ & 10,9 & $3.6 \pm 0.1$ & 14 \\
\hline
\end{tabular}

aThe 2MASS source name includes the J2000 sexagesimal, equatorial position in the form: hhmmssss+ddmmsss (Cutri et al. 2003).

${ }^{\mathrm{b}}$ Stellar heliocentric radial velocity

${ }^{*}$ These stars are part of multiple stellar systems. HD 34700A is a spectroscopic binary and has two visual companions (Sterzik et al. 2005). CS Cha was recently found to be a long-period spectroscopic binary (Guenther et al. 2007). VW Cha has a visual companion at $\sim 0.7^{\prime \prime}$ (Brandner et al. 1996).

References. - (1) Sterzik et al. 2005; (2) Weintraub et al. 2000; (3) Barrado Y Navascués 2006; (4) Joergens \& Guenther 2001; (5) Guenther et al. 2007; (6) Luhman 2008; (7) van den Ancker et al. 1998; (8) Melo 2003; (9) Hughes et al. 1994; (10) Graham \& Heyer 1988; (11) this work, line fluxes with errorbars; (12) Ratzka et al. 2007, second entry; (13) Espaillat et al. 2007, second entry; (14) Lahuis et al. 2007 
Table 2. Summary of the observations. The observing time (in U.T.), the airmass, and the heliocentric radial velocity corrections $\left(\mathrm{v}_{\text {helio }}\right.$ ) are given at the beginning and at the end of the observations.

\begin{tabular}{|c|c|c|c|c|c|c|c|c|}
\hline Source & $\begin{array}{c}\text { Date } \\
\text { yyyy-mm-dd }\end{array}$ & $\begin{array}{c}\text { U.T. } \\
\text { hh:mm }\end{array}$ & $\begin{array}{l}\mathrm{t}_{\exp } \\
(\mathrm{s})\end{array}$ & Airmass & $\begin{array}{c}\text { Vhelio } \\
(\mathrm{km} / \mathrm{s})\end{array}$ & Calibrator & $\begin{array}{l}\mathrm{t}_{\exp } \\
(\mathrm{s})\end{array}$ & Airmass \\
\hline TW Hya & $2008-02-20$ & 02:37/04:07 & 3600 & $1.3 / 1.1$ & $13.16 / 13.03$ & HD 90957(K5III) & 240 & $1.0(\mathrm{~F})$ \\
\hline CS Cha & $2008-02-20$ & 05:00/06:29 & 3600 & $1.7 / 1.7$ & $12.01 / 11.97$ & BV441(M0III) & & \\
\hline VW Cha & $2008-02-20$ & $07: 07 / 07: 15$ & 3480 & $1.7 / 1.9$ & $12.06 / 12.03$ & HD 92682(K3II) & 240 & $1.6(\mathrm{P})$ \\
\hline VW Cha & 2008-02-20 & 07:35/09:01 & 3480 & $1.7 / 1.9$ & $12.06 / 12.03$ & HD 105340(K2II) & 240 & $1.7(\mathrm{~F})$ \\
\hline HD 34700A & 2008-02-21 & $01: 22 / 02: 51$ & 3600 & $1.2 / 1.6$ & $-27.40 /-27.55$ & EPS Tau(G9.5III) & 240 & $1.6(\mathrm{P})$ \\
\hline \multirow[t]{2}{*}{$\mathrm{T}$ Cha } & 2008-02-21 & 03:34/06:37 & 7200 & $1.9 / 1.7$ & $12.99 / 12.94$ & BV441(M0III) & 240 & $1.8(\mathrm{P})$ \\
\hline & & & & & & HD 105340(K2II) & 360 & $1.6(\mathrm{~F})$ \\
\hline \multirow[t]{2}{*}{ Sz 102} & 2008-02-21 & 07:41/09:11 & 3600 & $1.3 / 1.1$ & $28.74 / 28.64$ & HD 136422(K5III) & 360 & $1.2(\mathrm{P})$ \\
\hline & & & & & & HD 139127 & 600 & $1.1(\mathrm{~F})$ \\
\hline CS Cha & $2008-02-22$ & 02:23/04:08 & 4140 & $1.9 / 1.7$ & $12.03 / 12.0$ & BV441(M0III) & 240 & $1.9(\mathrm{P})$ \\
\hline CS Cha & $2008-02-22$ & 04:28/06:00 & 3600 & $1.7 / 1.7$ & $11.99 / 11.95$ & HD 92682(K3II) & 360 & $1.6(\mathrm{~F})$ \\
\hline \multirow[t]{2}{*}{ Sz 73} & $2008-02-22$ & 06:58/09:17 & 5400 & $1.4 / 1.0$ & $29.36 / 29.19$ & HD 136422(K5III) & 360 & $1.4(\mathrm{P})$ \\
\hline & & & & & & HD 139127(K4.5III) & 600 & $1.1(\mathrm{~F})$ \\
\hline
\end{tabular}


Table 3. Summary of the VISIR results. For [Ne II] detections we assume a Gaussian for the line profile and a first order polynomial for the continuum. The last column gives the velocity of the peak emission in the stellocentric frame.

\begin{tabular}{lcccc}
\hline \hline Source & $\begin{array}{c}\text { [Ne II }] \\
\text { detection? }\end{array}$ & $\begin{array}{c}\text { Line Flux* } \\
\left(\times 10^{-14} \mathrm{erg} \mathrm{cm}^{-2} \mathrm{~s}^{-1}\right)\end{array}$ & $\begin{array}{c}\text { FWHM } \\
(\mathrm{km} / \mathrm{s})\end{array}$ & $\begin{array}{c}\mathrm{v}_{\text {peak }} \\
(\mathrm{km} / \mathrm{s})\end{array}$ \\
\hline HD 34700A & $\mathrm{N}$ & $<0.5$ & & \\
TW Hya & $\mathrm{Y}$ & $3.8 \pm 0.3$ & $14.6 \pm 0.7$ & $-6.2 \pm 0.3$ \\
CS Cha & $\mathrm{Y}^{\mathrm{a}}$ & $2.3 \pm 0.2$ & $27 \pm 2$ & $-3.3 \pm 0.7$ \\
VW Cha & $\mathrm{N}$ & $<0.6$ & & \\
T Cha & $\mathrm{Y}$ & $2.2 \pm 0.3$ & $42 \pm 4$ & $-4 \pm 2$ \\
Sz 73 & $\mathrm{Y}$ & $1.1 \pm 0.2$ & $60 \pm 8$ & $-99 \pm 3$ \\
Sz 102 & $\mathrm{N}$ & $<0.5$ & & \\
\hline
\end{tabular}

*In the case of non-detections we provide $3 \sigma$ upper limits to the line flux assuming a width of $10 \mathrm{~km} / \mathrm{s}$, equal to the instrument resolution. If the line is broader the flux upper limit would increase proportionally with the line FWHM by the factor (FWHM/10).

${ }^{\text {a }}$ In this case the continuum emission is not detected 
Table 4. Stellar and disk parameters used to generate the [Ne II] profiles in Fig. 4. Stellar ionizing flux $\Phi$ were fixed to $10^{41}$ photons/s. The last column provides the ratio between the observed and predicted [Ne II] line luminosity, which is also the scaling factor for the ionizing flux $\Phi$. The comparison between models and observations yields $\Phi=2.5,13$, and $1.9 \times 10^{41}$ photons/s for TW Hya, CS Cha, and T Cha respectively with an unceratinty of a factor of 2-3.

\begin{tabular}{lccccc}
\hline \hline Source & $\begin{array}{c}\mathrm{M}_{\star} \\
\left(\mathrm{M}_{\odot}\right)\end{array}$ & $\begin{array}{c}\text { Disk inclination } \\
\left({ }^{\circ}\right)\end{array}$ & $\begin{array}{c}\text { Distance } \\
(\mathrm{pc})\end{array}$ & Ref. & $\mathrm{L}_{\mathrm{obs}} / \mathrm{L}_{\text {mod }}$ \\
\hline TW Hya & 0.7 & 4 & 51 & $1,2,3$ & 2.5 \\
CS Cha & 0.9 & $45^{*}$ & 160 & 4,5 & 13 \\
T Cha & 1.5 & 75 & 66 & 6,7 & 1.9 \\
\hline
\end{tabular}

${ }^{*}$ Chosen to provide a good match to the [Ne II] line centroid and FWHM

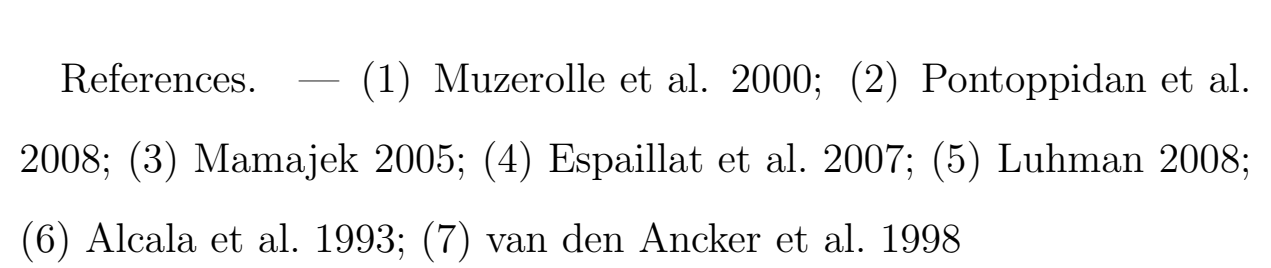

